\title{
AN APPROACH TO GROUP DECISION MAKING BASED ON 2-DIMENSION UNCERTAIN LINGUISTIC INFORMATION
}

\author{
Peide Liu \\ School of Management Science and Engineering, Shandong University of Finance and Economics, \\ 250014 Jinan Shandong, China \\ E-mail:peide.liu@gmail.com
}

Received 29 May 2011; accepted 18 September 2011

\begin{abstract}
Based on the definition of 2-dimension linguistic information of multiple attribute decision making problems proposed by Zhu, Zhou and Yang (2009), the information on evaluation is extended to 2-dimension uncertain linguistic variables, and a new method is proposed to solve the multiple-attribute group decision making problems in which the attribute values take the form of 2-dimension uncertain linguistic variables and the attribute weights are unknown. Firstly, the II class of uncertain linguistic information is transformed into the subjective weights of the experts, and then the subjective weights, the similarity degree of experts' evaluation information and authority weights are aggregated to the comprehensive weights of each expert. By the comprehensive weights, the group decision making matrix is produced by weighting evaluation information of each expert. Then the maximum deviation method is used to calculate the attribute weights and TOPSIS method is proposed to rank the alternatives. Finally, an example is given to illustrate the decision-making steps and the effectiveness of this method.
\end{abstract}

Keywords: 2-dimension uncertain linguistic variables, multiple attribute group decision making, TOPSIS.

Reference to this paper should be made as follows: Liu, P. 2012. An approach to group decision making based on 2-dimension uncertain linguistic information, Technological and Economic Development of Economy 18(3): 424-437.

JEL Classifcation: D81.

\section{Introduction}

Multiple attribute decision making (MADM) has been extensively applied to various areas ranging from economics to engineering technology. However, for some decision-making problems, such as personality assessment, automotive performance evaluation, etc. (Yan et 
al. 2011; Han, Liu 2011; Kaya, Kahraman 2011), the decision makers often give the evaluation information as the linguistic terms directly, such as good, medium good, medium, medium poor and poor, etc. For example, when we want to make a decision-making about buying a car, usually, we may consider the price, performance, appearance, comfort, etc. Except that the price can be expressed by quantitative data, but for performance, appearance and comfort we can give the evaluation information by the linguistic terms. For instance, we can think that a car's performance is good, and another's is medium. Here, "good" and "medium" belong to the linguistic terms. If the decision making environment is more fuzzy and uncertain, the decision makers also use the interval linguistic value or the uncertain linguistic to express the evaluation information. Some achievements have been made in the study of the MADM problems based on the uncertain linguistic variables (Zhang et al. 2006, 2007; Cheng et al. 2006; Xu 2006c, 2006d; Xu et al. 2007; Wei et al. 2007; Liu 2011; Liu, Su 2010; Liu, Wang 2011; Liu, Zhang 2010, 2011a, 2011b; Liu et al. 2011). For example, Zhang et al. (2007) studied the MADM problems in which the attribute value is the uncertain linguistic variables and the attribute weight is known, and proposed the UEWAA operator to aggregate the group decision making information and utilized the probability matrix ranking formula to rank the order of the alternatives; Zhang et al. (2006) studied the multiple attribute group decision making problems in which the attribute values and attribute weights also take the form of linguistic information, and proposed the ULWM operator to aggregate the comprehensive attribute values of the alternatives and ranked the alternatives based on ranking the fuzzy complementary judgment matrix. Cheng et al. (2006) studied the multiple attribute decision making problems in which the information on evaluation is the uncertain linguistic information and the decision making matrix has some unknown values, and proposed a method which can fill the unknown values in linguistic decision making matrix and a method which can determine the comprehensive attribute weights based on attribute subjective and objective weights. Xu et al. (2007) studied the multiple attribute decision making problems in which the attribute value is the uncertain linguistic variables and the attribute weight is unknown, and proposed the projection model to determine the attribute weigh, and then utilized the probability matrix ranking formula to rank the alternatives. Xu (2008b) investigated group decision making problems with multiple types of linguistic preference relations, and proposed a method to reach consensus among the individual preferences and the group's opinion. Wei et al. (2007) studied the multiple attribute group decision making problems with uncertain linguistic information, in which the attribute weight and expert weight take the form of real numbers, and the preference value takes the form of uncertain linguistic variables, and proposed a new method based on the ULWGM and the ULHGA operators. Xu (2009) defined some unbalanced linguistic label sets, and then developed some transformational functions to unify the given multigranular linguistic labels in a unique linguistic label set without lossing the information. Moreover, he utilized the uncertain linguistic weighted averaging operator to aggregate all individual uncertain linguistic decision matrices into a collective one, and defined two measures for similarity: one for measuring the similarity degree between each pair of uncertain linguistic variables, and the other for checking the degrees of consensus among the individual uncertain linguistic decision matrices and the collective uncertain linguistic decision matrix. Finally, an interactive approach to MAGDM with multigranular uncertain linguistic information was proposed. $\mathrm{Xu}(2010)$ proposed that 
the uncertain linguistic weighted geometric mean operator is utilized to aggregate all the individual uncertain multiplicative linguistic preference relations into a collective one, and then a simple approach is developed to determine the experts' weights by utilizing the consensus degrees among the individual uncertain multiplicative linguistic preference relations and the collective uncertain multiplicative linguistic preference relations. Furthermore, a proposal for a practical interactive procedure for group decision making is given based on uncertain multiplicative linguistic preference relations.

There are another decision making problems in the real decision making situation, for example, the evaluation on the projects or awards for the science and technology, the peer review of the master and doctor thesis or papers, etc. The decision makers not only make the conclusions on evaluation, but also show the evaluation reliability of themselves with the familiarity and other forms. For such decision making problems, Zhu et al. (2009) proposed the definition of the 2-dimension linguistic information to use I and II class of the linguistic evaluation information to describe the evaluation of decision makers with respect to the evaluation objects, where the I class of the linguistic evaluation information is used to describe the decision making objects, and the II class is used to describe the subjective evaluation of the reliability of the decision results. Finally, a group decision making method based on the evidence combination rules is given. However, the method proposed by Zhu et al. (2009) was meant to deal with single attribute decision making problems, but not to solve the multiple attribute problems, and the decision making method was more complex.

Based on the definition of 2-dimension linguistic information proposed by Zhu et al. (2009), the evaluation information is extended to 2-dimension uncertain linguistic variables and multiple attribute decision making problems, and a new method is proposed to solve the multiple attribute group decision making problems in which the attribute values take the form of 2-dimension uncertain linguistic variables and the attribute weight is unknown. Firstly, the II class of uncertain linguistic information is transformed into the subjective weight of the experts, and the similarity degree of experts' evaluation information and authority weights of each expert are aggregated to the comprehensive weights of each expert in different attributes and alternatives, the comprehensive weights can be used to aggregate each expert's evaluation information into the group decision making matrix. Then, the maximum deviation method is used to calculate the attribute weights, and TOPSIS method is proposed to rank the alternatives. Finally, an example is given to illustrate the decision-making steps.

\section{The description and the operation rules of the uncertain linguistic information}

Support that $S=\left(s_{0}, s_{1}, \cdots, s_{l-1}\right)$ is a pre-defined and ordered linguistic term set with odd elements, and $S$ should satisfy the following properties (Xu 2004a, 2004b; Xu 2006a):

1. The set is ordered: $s_{i} \prec s_{j}$, if and only if $i<j$,

2. There is the negation operator: $\operatorname{neg}\left(s_{i}\right)=s_{l-i}$,

3. Maximum operator: $\max \left(s_{i}, s_{j}\right)=s_{i}$, if $i \geq j$,

4. Minimum operator: $\min \left(s_{i}, s_{j}\right)=s_{i}$, if $i \leq j$. 
In practice, let $l$ be equal to $3,5,7,9$, etc. it can be defined as:

$S=\left(s_{0}, s_{1}, s_{2}\right)=$ (poor, fair, good).

$S=\left(s_{0}, s_{1}, s_{2}, s_{3}, s_{4}\right)=$ (very poor, poor, fair, good, very good).

$S=\left(s_{0}, s_{1}, s_{2}, s_{3}, s_{4}, s_{5}, s_{6}\right)=$ (very poor, poor, slightly poor, fair, slightly good, good, very good).

$S=\left(s_{0}, s_{1}, s_{2}, s_{3}, s_{4}, s_{5}, s_{6}, s_{7}, s_{8}\right)=$ (extremely poor, very poor, poor, slightly poor, fair, slightly good, good, very good, extremely good).

In the process of information aggregation, however, some results may not exactly match any linguistic labels in $S$. To preserve all the given information, the discrete term set $S$ is extended to a continuous term set $\bar{S}=\left\{s_{\alpha} \mid \alpha \in[0, q]\right\}$, where $s_{\alpha}$ meets all the characteristics above and $q(q \succ l)$ is a sufficiently large positive integer. If $s_{\alpha} \in S$, then we call $s_{\alpha}$ the original term, otherwise, we call $s_{\alpha}$ the virtual term. In general, the decision maker uses the original linguistic terms to evaluate alternatives, and the virtual linguistic terms can only appear in calculation and ranking (Xu 2004b, 2004c).

Definition 1 (Xu 2006a): Let $\tilde{s}=\left[s_{a}, s_{b}\right]$, where $s_{a}, s_{b} \in \bar{S}$ and $a \leq b$, and $s_{a}$ and $s_{b}$ are the lower and the upper limits respectively, then we call $\tilde{s}$ an uncertain linguistic variable.

Suppose that the set $\tilde{S}$ is composed of all the uncertain linguistic variables, and $\tilde{s}_{1}=\left[s_{a 1}, s_{b 1}\right]$ and $\tilde{s}_{2}=\left[s_{a 2}, s_{b 2}\right]$ are any two uncertain linguistic variables, and $\lambda_{1} \in[0,1]$ and $\lambda_{1} \in[0,1]$, then their operation rules are shown as follows (Xu 2004b, 2006a):

1. $\tilde{s}_{1} \oplus \tilde{s}_{2}=\left[s_{a 1}, s_{b 1}\right] \oplus\left[s_{a 2}, s_{b 2}\right]=\left[s_{a 1+a 2}, s_{b 1+b 2}\right]$,

2. $\tilde{s}_{1} \otimes \tilde{s}_{2}=\left[s_{a 1}, s_{b 1}\right] \otimes\left[s_{a 2}, s_{b 2}\right]=\left[s_{a 1 \times a 2}, s_{b 1 \times b 2}\right]$,

3. $\tilde{s}_{1} / \tilde{s}_{2}=\left[s_{a 1}, s_{b 1}\right] /\left[s_{a 2}, s_{b 2}\right]=\left[s_{a 1 / b 2}, s_{b 1 / a 2}\right]$, if $a 2 \neq 0, b 2 \neq 0$,

4. $\lambda \tilde{s}_{1}=\lambda\left[s_{a 1}, s_{b 1}\right]=\left[s_{\lambda * a 1}, s_{\lambda^{*} b 1}\right]$,

5. $\lambda\left(\tilde{s}_{1} \oplus \tilde{s}_{2}\right)=\lambda \tilde{s}_{1} \oplus \lambda \tilde{s}_{2}$,

6. $\left(\lambda_{1}+\lambda_{2}\right) \tilde{s}_{1}=\lambda_{1} \tilde{s}_{1} \oplus \lambda_{2} \tilde{s}_{1}$.

Definition 2 (Xu 2006b; Liu, Zhang 2009): Let $\tilde{s}_{1}=\left[s_{a 1}, s_{b 1}\right]$ and $\tilde{s}_{2}=\left[s_{a 2}, s_{b 2}\right]$ be any two uncertain linguistic variables, then the distance of the $\tilde{s}_{1}$ and $\tilde{s}_{2}$ is defined as follows:

$$
d\left(\tilde{s}_{1}, \tilde{s}_{2}\right)=\sqrt{(a 2-a 1)^{2}+(b 2-b 1)^{2} / 2} .
$$

Definition $3(\mathrm{Xu} 2005,2009)$ : If $\tilde{s}_{1}=\left[s_{a 1}, s_{b 1}\right]$ and $\tilde{s}_{2}=\left[s_{a 2}, s_{b 2}\right]$ are any two uncertain linguistic variables, and $l$ is the number of the linguistic variables in the linguistic variables set $S$, then the similarity degree of the $\tilde{s}_{1}$ and $\tilde{s}_{2}$ is defined as follows:

$$
s\left(\tilde{s}_{1}, \tilde{s}_{2}\right)=1-\frac{a b s(a 1-a 2)+a b s(b 1-b 2)}{2 \times(l-1)} .
$$

\section{The decision making method}

\subsection{The description of the decision making problems}

If $A=\left(a_{1}, a_{2}, \cdots, a_{m}\right)$ is the set of alternatives, and $C=\left(c_{1}, c_{2}, \cdots, c_{n}\right)$ is the set of attributes. $W=\left(w_{1}, w_{2}, \ldots, w_{n}\right)$ is the weight vector of attributes $c_{j}(j=1,2, \cdots, n)$ and it is unknown, but 
meets $0 \leq w_{j} \leq 1, \sum_{j=1}^{n} w_{j}=1$. If $\left(e_{1}, e_{2}, \cdots, e_{p}\right)$ is the experts set in the group decision making, and $\lambda=\left(\lambda_{1}, \lambda_{2}, \cdots, \lambda_{p}\right)$ is the authority weight set of the experts, where $0 \leq \lambda_{k} \leq 1, \sum_{k=1}^{p} \lambda_{k}=1$. Suppose that $\left(\left[x_{i j}^{L k}, x_{i j}^{U k}\right],\left[g_{i j}^{L k}, g_{i j}^{U k}\right]\right)$ is the attribute value of the attribute $c_{j}$ in the alternative $a_{i}$, given by the expert $e_{k}$, which takes the form of the 2-dimension uncertain linguistic information, where $\left[x_{i j}^{L k}, x_{i j}^{U k}\right]$ is the I class of the uncertain linguistic evaluation information, and it shows the object evaluation given by the expert, and $x_{i j}^{L k}$ and $x_{i j}^{U k}$ are the elements of the pre-defining linguistic evaluation set $S_{1}=\left(s_{0}, s_{1}, \cdots, s_{l-1}\right)$, and $\left[g_{i j}^{L k}, g_{i j}^{U k}\right]$ is the II class of the uncertain linguistic evaluation information, and it shows the subjective evaluation of the reliability of the decision results, and $g_{i j}^{L k}$ and $g_{i j}^{U k}$ are the element of the pre-defining linguistic evaluation set $S_{2}=\left(s_{0}, s_{1}, \cdots, s_{t-1}\right)$. Based on these conditions, the order of the alternatives of multiple attribute decision making problems based on the 2-dimension uncertain linguistic variables can be ranked.

\subsection{The decision making method}

\subsubsection{Transform the II class of the uncertain linguistic evaluation information to the subjective weight of the decision makers}

The II class of the uncertain linguistic evaluation information shows the subjective evaluation of the reliability of the decision results. No one knows the reliability of the subjective evaluation better than the decision makers themselves, under the premise that the rational, knowledge and experience level of the decision makers can satisfy the requirement of the decision making. Therefore, we can transform the II class of the uncertain linguistic evaluation information, given by the decision makers, to the subjective weight of the decision makers. The larger the evaluation value of the II class of the linguistic evaluation information is (the more faith the decision maker has), and the larger the weight of decision maker is, and vice versa.

\section{The UL-OWA operator}

Definition 4 (Yager 2004): Let function $\rho:[0,1] \rightarrow[0,1]$ satisfy:

1. $\rho(0)=0$;

2. $\rho(1)=1$;

3. if $x>y$, then $\rho(x)>\rho(y)$.

Then $\rho$ is called the basic unit-interval monotonic function (the BUM function).

Definition 5 (Zhang, Xu 2005): If $\left[s_{a}, s_{b}\right]$ is the uncertain linguistic variable, and

$$
f_{\rho}\left(\left[s_{a}, s_{b}\right]\right)=s_{0}^{1} \frac{d \rho(y)}{d y}(b-y(b-a)) d y,
$$

then $f$ is called the uncertain linguistic variable OWA operator (the UL-OWA operator).

$$
\text { If } \rho(y)=y^{\delta}(\delta \geq 0) \text {, then } f_{\rho}\left(\left[s_{a}, s_{b}\right]\right)=\frac{s_{b+\delta a}}{\delta+1} \text {. }
$$


2. Transform the II class of the uncertain linguistic evaluation information to the linguistic information

We utilize the UL-OWA operator to transform the II class of the uncertain linguistic variable $\left[g_{i j}^{L k}, g_{i j}^{U k}\right]$ to the linguistic variable $g_{i j}^{k}=f_{\rho}\left[g_{i j}^{L k}, g_{i j}^{U k}\right]$.

3. Calculate the weight $\xi_{i j}^{k}$ of the attribute $c_{j}$ under the alternative $a_{i}$ given by the expert $e_{k}$

$$
\xi_{i j}^{k}=\frac{g_{i j}^{k}}{\sum_{j=1}^{n} g_{i j}^{k}} .
$$

\subsubsection{Calculate the relative similarity degree of decision making information of the experts}

First, we should calculate the relative similarity degree of the evaluation value of the attribute $c_{j}$ in the alternative $a_{i}$, given by any two experts $e_{k}$ and $e_{q}$. The formula (8) can be used to calculate the relative similarity degree $S_{i j}(k, q)$ of any two uncertain linguistic variables $\left[x_{i j}^{L k}, x_{i j}^{U k}\right]$ and $\left[x_{i j}^{L q}, x_{i j}^{U q}\right]$ given by the experts $e_{k}$ and $e_{q}$. Then we should calculate the average similarity degree in the expert group of the evaluation value of the attribute $c_{j}$ in the alternative $a_{i}$, given by the expert $e_{k}$ :

$$
A S_{i j}(k)=\frac{1}{p-1} \sum_{q=1, q \neq k}^{p} S_{i j}(k, q) .
$$

Based on these, we can calculate the relative similarity degree in the expert group of the evaluation value of the attribute $c_{j}$ in the alternative $a_{i}$, given by the expert $e_{k}$ :

$$
R S_{i j}(k)=\frac{A S_{i j}(k)}{\sum_{k=1}^{p} A S_{i j}(k)} \text {. }
$$

\subsubsection{Determine the comprehensive weight of experts}

We should comprehensively consider these conditions, such as the similarity degree of the expert evaluation information and the expert group evaluation information, the weight transformed by the II class of the uncertain linguistic evaluation information and the authority weight of the experts.

The multiplication synthesis method has the multiplier effect (Zeng 1997). So we aggregate the similarity degree of the expert evaluation information and the expert group evaluation information, and the weight transformed by the II class of the uncertain linguistic evaluation information, then we can get the comprehensive weight.

$$
\gamma_{i j}^{k}=\frac{\xi_{i j}^{k} \times R S_{i j}(k)}{\sum_{k=1}^{p} \xi_{i j}^{k} \times R S_{i j}(k)}
$$


We further aggregate the authority weight of the experts, and we finally get the comprehensive weight of the attribute $c_{j}$ in the alternative $a_{i}$, given by the expert $e_{k}$.

$$
\omega_{i j}^{k}=\alpha \times \lambda_{k}+(1-\alpha) \times \gamma_{i j}^{k},
$$

where $\alpha$ is the weight coefficient, and $0 \leq \alpha \leq 1$. The value of $\alpha$ shows the preference of the expert.

\subsubsection{Aggregate the expert's evaluation information}

We should aggregate the expert's evaluation information, and transform the 2-dimension linguistic evaluation information into 1-dimension linguistic evaluation information $Z=\left[z_{i j}\right]_{m \times n}$, where $z_{i j}=\left[z_{i j}^{L}, z_{i j}^{U}\right]$, then:

$$
z_{i j}^{L}=\sum_{k=1}^{p}\left(\omega_{i j}^{k} \times x_{i j}^{L k}\right), z_{i j}^{U}=\sum_{k=1}^{p}\left(\omega_{i j}^{k} \times x_{i j}^{U k}\right) .
$$

\subsubsection{Calculate the attribute weight based on the maximizing deviations method}

The attribute weight is unknown, and the uncertainty of the attribute weight will result in the uncertainty of the ranking order of the alternatives. In general, if the attribute value $z_{i j}(j=1,2, \cdots, n)$ among all the alternatives are little different with respect to attribute $c_{j}$, it shows that the attribute $c_{j}$ plays a less important role in the decision making procedure, and the smaller weight will be given. Contrariwise, if the attribute $c_{j}$ makes the attribute values $z_{i j}(j=1,2, \cdots, n)$ among all the alternatives have obvious differences, such an attribute plays an important role in choosing the best alternative. Therefore, while ranking the alternatives, the larger the deviation of the attribute value of the alternative is, the larger its weight is, and vice versa (Zhou, Liu 2007).

For the attribute $c_{j}$, the deviation value of alternative $A_{i}$ to all the other alternatives can be defined as $D_{i j}\left(w_{j}\right)=\sum_{l=1}^{m} d\left(z_{i j}, z_{l j}\right) w_{j}$, then $D_{j}\left(w_{j}\right)=\sum_{i=1}^{m} D_{i j}\left(w_{j}\right)=\sum_{i=1}^{m} \sum_{l=1}^{m} d\left(z_{i j}, z_{l j}\right) w_{j}$ and represents the total deviation value of all alternatives to the other alternatives for the attribute $c_{j} . D\left(w_{j}\right)=\sum_{j=1}^{n} D_{j}\left(w_{j}\right)=\sum_{j=1}^{n} \sum_{i=1}^{m} \sum_{l=1}^{m} d\left(z_{i j}, z_{l j}\right) w_{j}$ represents the deviation of all attributes to all alternatives. The optimization model is constructed as follows (Xu 2008a):

$$
\left\{\begin{array}{l}
\max D\left(w_{j}\right)=\sum_{j=1}^{n} \sum_{i=1}^{m} \sum_{l=1}^{m} d\left(z_{i j}, z_{l j}\right) w_{j} \\
\text { s.t } \sum_{j=1}^{n} w_{j}^{2}=1, w_{j} \geq 0, j=1,2 \ldots \ldots n
\end{array} .\right.
$$


We can get the normalized attribute weight based on this model:

$$
w_{j}=\frac{\sum_{i=1}^{m} \sum_{l=1}^{m} d\left(z_{i j}, z_{l j}\right)}{\sum_{j=1}^{n} \sum_{i=1}^{m} \sum_{l=1}^{m} d\left(z_{i j}, z_{l j}\right)} .
$$

\subsubsection{Determine the order of the alternatives based on TOPSIS}

TOPSIS (Technique for Order Performance by Similarity to Ideal Solution) method is the famous multiple attribute decision making method, proposed by the Hwang and Yoon (1981). This method is usually used to solve such multiple attribute decision making problems when the attribute value is the real number. This paper utilizes the TOPSIS method to solve the multiple attribute decision making problems in which the attribute value is the uncertain linguistic variables, based on the distance formula between two uncertain linguistic variables.

1. Calculate the weight matrix:

$$
V=\left[v_{i j}\right]_{m \times n}=\left[\begin{array}{cccc}
{\left[v_{11}^{L}, v_{11}^{U}\right]} & {\left[v_{12}^{L}, v_{12}^{U}\right]} & \cdots & {\left[v_{1 n}^{L}, v_{1 n}^{U}\right]} \\
{\left[v_{21}^{L}, v_{21}^{U}\right]} & {\left[v_{22}^{L}, v_{22}^{U}\right]} & \cdots & {\left[v_{2 n}^{L}, v_{2 n}^{U}\right]} \\
\vdots & \cdots & \cdots & \vdots \\
{\left[v_{m 1}^{L}, v_{m 1}^{U}\right]} & {\left[v_{m 2}^{L}, v_{m 2}^{U}\right]} & \cdots & {\left[v_{m n}^{L}, v_{m n}^{U}\right]}
\end{array}\right],
$$

where

$$
v_{i j}^{L}=w_{j} z_{i j}^{L}, v_{i j}^{U}=w_{j} z_{i j}^{U} .
$$

2. Calculate the positive and negative ideal solution of the weighted matrix:

$$
\begin{aligned}
& V^{+}=\left(v_{1}^{+}, v_{2}^{+}, \cdots, v_{n}^{+}\right)=\left(\left[v_{1}^{L+}, v_{1}^{U+}\right],\left[v_{2}^{L+}, v_{2}^{U+}\right], \cdots,\left[v_{n}^{L+}, v_{n}^{U+}\right]\right), \\
& V^{-}=\left(v_{1}^{-}, v_{2}^{-}, \cdots, v_{n}^{-}\right)=\left(\left[v_{1}^{L-}, v_{1}^{U-}\right],\left[v_{2}^{L-}, v_{2}^{U-}\right], \cdots,\left[v_{n}^{L-}, v_{n}^{U-}\right]\right),
\end{aligned}
$$

where

$$
v_{j}^{L+}=\max _{i}\left(v_{i j}^{L}\right), v_{j}^{U+}=\max _{i}\left(v_{i j}^{U}\right), v_{j}^{L-}=\min _{i}\left(v_{i j}^{L}\right), v_{j}^{U-}=\min _{i}\left(v_{i j}^{U}\right) .
$$

3. Calculate the distance between each alternative and the positive and negative ideal solution:

$$
D^{+}=\left(d_{1}^{+}, d_{2}^{+}, \cdots, d_{m}^{+}\right) \text {and } D^{-}=\left(d_{1}^{-}, d_{2}^{-}, \cdots, d_{m}^{-}\right)
$$

where

$$
\left\{\begin{array}{l}
d_{i}^{+}=\left[\sum_{j=1}^{n}\left(d\left(v_{i j}, v_{j}^{+}\right)\right)^{2}\right]^{1 / 2} \\
d_{i}^{-}=\left[\sum_{j=1}^{n}\left(d\left(v_{i j}, v_{j}^{-}\right)\right)^{2}\right]^{1 / 2}
\end{array}\right.
$$


where $d\left(v_{i j}, v_{j}^{+}\right)$is the distance between the uncertain linguistic variables $v_{i j}$ and $v_{j}^{+}, d\left(v_{i j}, v_{j}^{-}\right)$ is the distance between the uncertain linguistic variables $v_{i j}$ and $v_{j}^{-}$. They can be calculated by the formula (7).

4. Calculate the relative closeness degree of each alternative.

Let $C=\left(c_{1}, c_{2}, \cdots, c_{m}\right)$ be the relative closeness degree of each alternative, where

$$
c_{i}=\frac{d_{i}^{-}}{d_{i}^{+}+d_{i}^{-}} \quad(i=1,2, \cdots, m) .
$$

5. Rank the order of the alternatives

We can rank the order of the alternatives based on the values of the relative closeness degree. The lager the relative closeness degree is, the better the alternative is, vice versa.

\section{An illustrate example}

A practical use of the proposed approach involves the technological innovation ability to evaluate the four enterprises $\left\{a_{1}, a_{2}, a_{3}, a_{4}\right\}$, the attributes are shown as follows: the ability of innovative resources input $\left(C_{1}\right)$, the ability of innovation management $\left(C_{2}\right)$, the ability of innovation tendency $\left(C_{3}\right)$ and the ability of research and development $\left(C_{4}\right)$. Based on the four attributes, the three experts $\left\{e_{1}, e_{2}, e_{3}\right\}$ evaluated the technological innovation ability of the four enterprises. Supposedly $\lambda=(0.4,0.32,0.28)$ is the weight vector of the three experts, and the attribute values given by the experts take the form of 2 -dimension uncertain linguistic variables, shown in Tables 1,2 and 3 . The experts utilize the I class of the linguistic evaluation set $S_{1}=\left(s_{0}, s_{1}, s_{2}, s_{3}, s_{4}, s_{5}, s_{6}\right)$ and the II class of the linguistic evaluation set $S_{2}=\left(s_{0}, s_{1}, s_{2}, s_{3}, s_{4}\right)$, and the attribute weight is unknown.

Table 1. The attributes' values with respect to four enterprises given by expert $e_{1}$

\begin{tabular}{ccccc}
\hline Enterprises & Attribute $\left(C_{1}\right)$ & Attribute $\left(C_{2}\right)$ & Attribute $\left(C_{3}\right)$ & Attribute $\left(C_{4}\right)$ \\
\hline$a_{1}$ & $\left(\left[s_{5}, s_{5}\right],\left[s_{2}, s_{3}\right]\right)$ & $\left(\left[s_{2}, s_{3}\right],\left[s_{3}, s_{3}\right]\right)$ & $\left(\left[s_{4}, s_{5}\right],\left[s_{4}, s_{4}\right]\right)$ & $\left(\left[s_{3}, s_{4}\right],\left[s_{1}, s_{2}\right]\right)$ \\
\hline$a_{2}$ & $\left(\left[s_{3}, s_{4}\right],\left[s_{2}, s_{3}\right]\right)$ & $\left(\left[s_{5}, s_{5}\right],\left[s_{3}, s_{3}\right]\right)$ & $\left(\left[s_{3}, s_{3}\right],\left[s_{4}, s_{4}\right]\right)$ & $\left(\left[s_{4}, s_{4}\right],\left[s_{1}, s_{2}\right]\right)$ \\
\hline$a_{3}$ & $\left(\left[s_{2}, s_{3}\right],\left[s_{2}, s_{3}\right]\right)$ & $\left(\left[s_{3}, s_{4}\right],\left[s_{3}, s_{3}\right]\right)$ & $\left(\left[s_{3}, s_{4}\right],\left[s_{4}, s_{4}\right]\right)$ & $\left(\left[s_{4}, s_{5}\right],\left[s_{1}, s_{2}\right]\right)$ \\
\hline$a_{4}$ & $\left(\left[s_{5}, s_{6}\right],\left[s_{2}, s_{3}\right]\right)$ & $\left(\left[s_{1}, s_{2}\right],\left[s_{3}, s_{3}\right]\right)$ & $\left(\left[s_{2}, s_{3}\right],\left[s_{4}, s_{4}\right]\right)$ & $\left(\left[s_{3}, s_{4}\right],\left[s_{1}, s_{2}\right]\right)$ \\
\hline
\end{tabular}

Table 2. The attributes' values with respect to four enterprises given by expert $e_{2}$

\begin{tabular}{ccccc}
\hline Enterprises & Attribute $\left(C_{1}\right)$ & Attribute $\left(C_{2}\right)$ & Attribute $\left(C_{3}\right)$ & Attribute $\left(C_{4}\right)$ \\
\hline$a_{1}$ & $\left(\left[s_{4}, s_{4}\right],\left[s_{3}, s_{4}\right]\right)$ & $\left(\left[s_{3}, s_{4}\right],\left[s_{2}, s_{3}\right]\right)$ & $\left(\left[s_{3}, s_{4}\right],\left[s_{3}, s_{3}\right]\right)$ & $\left(\left[s_{5}, s_{6}\right],\left[s_{3}, s_{4}\right]\right)$ \\
\hline$a_{2}$ & $\left(\left[s_{4}, s_{5}\right],\left[s_{3}, s_{4}\right]\right)$ & $\left(\left[s_{2}, s_{3}\right],\left[s_{2}, s_{3}\right]\right)$ & $\left(\left[s_{4}, s_{5}\right],\left[s_{3}, s_{3}\right]\right)$ & $\left(\left[s_{2}, s_{3}\right],\left[s_{3}, s_{4}\right]\right)$ \\
\hline
\end{tabular}


End of Table 2

\begin{tabular}{ccccc}
\hline Enterprises & Attribute $\left(C_{1}\right)$ & Attribute $\left(C_{2}\right)$ & Attribute $\left(C_{3}\right)$ & Attribute $\left(C_{4}\right)$ \\
\hline$a_{3}$ & $\left(\left[s_{3}, s_{4}\right],\left[s_{3}, s_{4}\right]\right)$ & $\left(\left[s_{4}, s_{4}\right],\left[s_{2}, s_{3}\right]\right)$ & $\left(\left[s_{2}, s_{3}\right],\left[s_{3}, s_{3}\right]\right)$ & $\left(\left[s_{3}, s_{4}\right],\left[s_{3}, s_{4}\right]\right)$ \\
\hline$a_{4}$ & $\left(\left[s_{5}, s_{5}\right],\left[s_{3}, s_{4}\right]\right)$ & $\left(\left[s_{4}, s_{5}\right],\left[s_{2}, s_{3}\right]\right)$ & $\left(\left[s_{1}, s_{2}\right],\left[s_{3}, s_{3}\right]\right)$ & $\left(\left[s_{4}, s_{4}\right],\left[s_{3}, s_{4}\right]\right)$ \\
\hline
\end{tabular}

Table 3. The attributes' values with respect to four enterprises given by expert $e_{3}$

\begin{tabular}{ccccc}
\hline Enterprises & Attribute $\left(C_{1}\right)$ & Attribute $\left(C_{2}\right)$ & Attribute $\left(C_{3}\right)$ & Attribute $\left(C_{4}\right)$ \\
\hline$a_{1}$ & $\left(\left[s_{5}, s_{5}\right],\left[s_{2}, s_{3}\right]\right)$ & $\left(\left[s_{3}, s_{3}\right],\left[s_{2}, s_{2}\right]\right)$ & $\left(\left[s_{4}, s_{4}\right],\left[s_{3}, s_{4}\right]\right)$ & $\left(\left[s_{4} s_{5}\right],\left[s_{1}, s_{1}\right]\right)$ \\
\hline$a_{2}$ & $\left(\left[s_{4}, s_{4}\right],\left[s_{2}, s_{3}\right]\right)$ & $\left(\left[s_{4}, s_{5}\right],\left[s_{2}, s_{2}\right]\right)$ & $\left(\left[s_{1}, s_{2}\right],\left[s_{3}, s_{4}\right]\right)$ & $\left(\left[s_{3}, s_{3}\right],\left[s_{1}, s_{1}\right]\right)$ \\
\hline$a_{3}$ & $\left(\left[s_{3}, s_{4}\right],\left[s_{2}, s_{3}\right]\right)$ & $\left(\left[s_{5}, s_{5}\right],\left[s_{2}, s_{2}\right]\right)$ & $\left(\left[s_{1}, s_{1}\right],\left[s_{3}, s_{4}\right]\right)$ & $\left(\left[s_{4}, s_{4}\right],\left[s_{1}, s_{1}\right]\right)$ \\
\hline$a_{4}$ & $\left(\left[s_{2}, s_{3}\right],\left[s_{2}, s_{3}\right]\right)$ & $\left(\left[s_{2}, s_{3}\right],\left[s_{2}, s_{2}\right]\right)$ & $\left(\left[s_{4}, s_{5}\right],\left[s_{3}, s_{4}\right]\right)$ & $\left(\left[s_{4}, s_{5}\right],\left[s_{1}, s_{1}\right]\right)$ \\
\hline
\end{tabular}

The evaluation steps used in this paper are proposed as follows:

\section{Transform the II class of the uncertain linguistic evaluation information to the subjective weight of the decision makers}

We select the BUM function $\rho(y)=y^{2}$, then the subjective weight is calculated as follows:

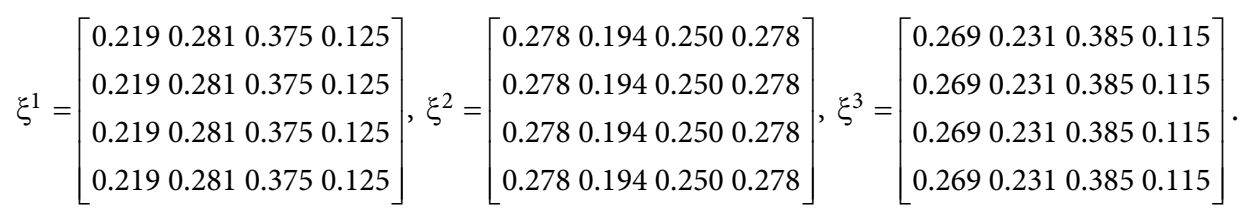

2. Calculate the relative similarity degree of the attribute $c_{j}$ under the alternative $a_{i}$ of each expert

$$
R S(1)=\left[\begin{array}{llll}
0.344 & 0.328 & 0.328 & 0.321 \\
0.328 & 0.346 & 0.375 & 0.317 \\
0.313 & 0.333 & 0.327 & 0.328 \\
0.354 & 0.333 & 0.375 & 0.328
\end{array}\right], R S(2)=\left[\begin{array}{llll}
0.313 & 0.328 & 0.328 & 0.321 \\
0.328 & 0.288 & 0.313 & 0.333 \\
0.344 & 0.350 & 0.365 & 0.328 \\
0.375 & 0.292 & 0.333 & 0.344
\end{array}\right], R S(3)=\left[\begin{array}{llll}
0.344 & 0.344 & 0.344 & 0.357 \\
0.344 & 0.365 & 0.313 & 0.350 \\
0.344 & 0.317 & 0.308 & 0.344 \\
0.271 & 0.375 & 0.292 & 0.328
\end{array}\right] \text {. }
$$

\section{Calculate the comprehensive weight}

Let the weight coefficient $\alpha=0.4$, then the comprehensive weights are:

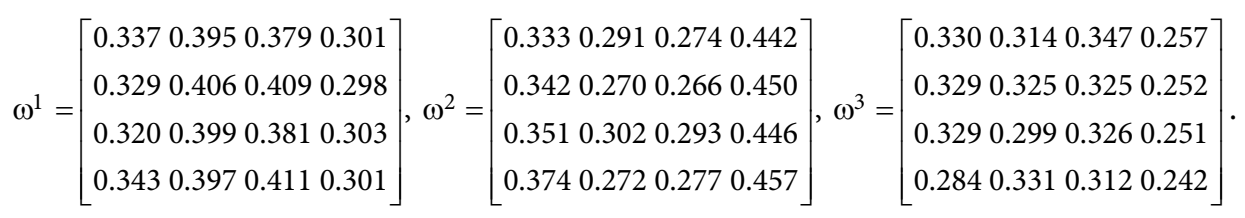


4. Transform the 2-dimension information into 1-dimension linguistic evaluation information and aggregate into the group decision matrix

$$
Z=\left[\begin{array}{llll}
{\left[s_{4.667}, s_{4.667}\right]} & {\left[s_{2.605}, s_{3.291}\right]} & {\left[s_{3.726}, s_{4.379}\right]} & {\left[s_{4.141}, s_{5.141}\right]} \\
{\left[s_{3.671}, s_{4.342}\right]} & {\left[s_{3.867}, s_{4.461}\right]} & {\left[s_{2.617}, s_{3.208}\right]} & {\left[s_{2.848}, s_{3.298}\right]} \\
{\left[s_{2.680}, s_{3.680}\right]} & {\left[s_{3.899}, s_{4.299}\right]} & {\left[s_{2.056}, s_{2.730}\right]} & {\left[s_{3.554}, s_{4.303}\right]} \\
{\left[s_{4.148}, s_{4.775}\right]} & {\left[s_{2.146}, s_{3.146}\right]} & {\left[s_{2.348}, s_{3.348}\right]} & {\left[s_{3.699}, s_{4.242}\right]}
\end{array}\right] .
$$

5. Calculate the attribute weight based on the maximizing deviations method

$$
W=\left(\begin{array}{llll}
0.248 & 0.275 & 0.246 & 0.231
\end{array}\right) .
$$

\section{Calculate the weighted decision making matrix}

$$
V=\left[\begin{array}{llll}
{\left[s_{1.158}, s_{1.158}\right]} & {\left[s_{0.716}, s_{0.905}\right]} & {\left[s_{0.917}, s_{1.078}\right]} & {\left[s_{0.954}, s_{1.185}\right]} \\
{\left[s_{0.911}, s_{1.078}\right]} & {\left[s_{1.064}, s_{1.227}\right]} & {\left[s_{0.644}, s_{0.790}\right]} & {\left[s_{0.656}, s_{0.760}\right]} \\
{\left[s_{0.665}, s_{0.913}\right]} & {\left[s_{1.073}, s_{1.182}\right]} & {\left[s_{0.506}, s_{0.672}\right]} & {\left[s_{0.819}, s_{0.992}\right]} \\
{\left[s_{1.030}, s_{1.185}\right]} & {\left[s_{0.590}, s_{0.865}\right]} & {\left[s_{0.578}, s_{0.824}\right]} & {\left[s_{0.853}, s_{0.978}\right]}
\end{array}\right] .
$$

7. Calculate the positive and negative ideal solution

$$
\begin{aligned}
& V^{+}=\left(\left[s_{1.158}, s_{1.185}\right]\left[s_{1.073}, s_{1.227}\right]\left[s_{0.917}, s_{1.078}\right]\left[s_{0.954}, s_{1.185}\right]\right), \\
& V^{-}=\left(\left[s_{0.665}, s_{0.913}\right]\left[s_{0.590}, s_{0.865}\right]\left[s_{0.506}, s_{0.672}\right]\left[s_{0.656}, s_{0.760}\right]\right) .
\end{aligned}
$$

8. Calculate the weight distance between each alternative and the positive and negative ideal solution

$$
\begin{aligned}
& D^{+}=\left(\begin{array}{llll}
0.340 & 0.500 & 0.595 & 0.554
\end{array}\right), \\
& D^{-}=\left(\begin{array}{llll}
0.680 & 0.488 & 0.455 & 0.401
\end{array}\right) .
\end{aligned}
$$

\section{Calculate the relative closeness degree}

\section{Rank the order of the alternatives}

$$
C=\left(\begin{array}{llll}
0.667 & 0.494 & 0.433 & 0.420
\end{array}\right) .
$$

Based on the value of the relative closeness degree, the order of each alternative is $a_{1} \succ a_{2} \succ a_{3} \succ a_{4}$.

In order to verify the effectiveness of the method, we use the method proposed by Zhu et al. (2009). However, the method is only used with the linguistic variables and single attribute decision making problems. In order to apply it, we had to covert the uncertain linguistic variables to linguistic variables by averaging algorithm, then calculate utility value for each attribute with respect to each alternative, and weight the utility value for all attributes and get an integrated utility value for each alternative. Finally, rank the alternatives by the integrated utility values.

The calculated ranking is $a_{1} \succ a_{2} \succ a_{3} \succ a_{4}$. There are the same results for two methods. 


\section{Conclusions}

Multiple attribute group decision making based on the 2-dimension uncertain linguistic variables are widely used in the real decision making. Firstly, the II class of uncertain linguistic information is transformed into the subjective weights of the experts, and the similarity degree of experts' evaluation information and authority weights of each expert are aggregated to the comprehensive weights of each expert in different attributes and alternatives, the comprehensive weights can be used to aggregate each expert' evaluation information into the group decision making matrix. Then, the maximum deviation method is used to calculate the attribute weights, and TOPSIS method is proposed to rank the alternatives. Finally, an illustrate example is given to show the decision-making steps and the effectiveness of this method. This method proposed in this paper is easy to use and understand, and it enriched and developed the theory and method of 2-dimension uncertain linguistic multiple attribute decision making, and it provided the new idea to solve the 2-dimension uncertain linguistic multiple attribute decision making. In the future, we shall continue working in the extension and application of the developed method to other domains.

\section{Acknowledgments}

This paper is supported by the National Natural Science Foundation of China (No. 71271124), the Humanities and Social Sciences Foundation of Ministry of Education of China (No. 10YJA630073 and 09YJA630088), and the Natural Science Foundation of Shandong Province (No. ZR2011FM036). The authors also would like to express appreciation to the anonymous reviewers for their very helpful comments that improved the paper.

\section{References}

Cheng, P. F.; Zhou, X. H.; Tang, X. P. 2006. A decision making method based on the uncertain linguistic, Statistics and Decision (24): 145-147.

Han, Z. S.; Liu, P. D. 2011. A fuzzy multi-attribute decision-making method under risk with unknown attribute weights, Technological and Economic Development of Economy 17(2): 246-258. http://dx.doi.org/10.3846/20294913.2011.580575

Hwang, C. L.; Yoon, K. S. 1981. Multiple Attribute Decision Making: Methods and Applications. Berlin: Springer-Verlag. http://dx.doi.org/10.1007/978-3-642-48318-9

Kaya, T.; Kahraman, C. 2011. A fuzzy approach to e-banking website quality assessment based on an integrated AHP-ELECTRE method, Technological and Economic Development of Economy 17(2):313-334. http://dx.doi.org/10.3846/20294913.2011.583727

Liu, P. D. 2011. A weighted aggregation operators multi-attribute group decision-making method based on interval-valued trapezoidal fuzzy numbers, Expert Systems with Applications 38(1): 1053-1060. http://dx.doi.org/10.1016/j.eswa.2010.07.144

Liu, P. D.; Su, Y. 2010. The multiple attribute decision making method based on the TFLHOWA operator, Computers and Mathematics with Applications 60(9): 2609-2615.

http://dx.doi.org/10.1016/j.camwa.2010.08.087 
Liu, P. D.; Wang, M. H. 2011. An extended VIKOR method for multiple attribute group decision making based on generalized interval-valued trapezoidal fuzzy numbers, Scientific Research and Essays 6(4): 760-765.

Liu, P. D.; Zhang, X. 2009. Multiple attribute decision making TOPSIS method based on the uncertain linguistic variables, Journal of Wuhan University of Technology 31(16): 158-161.

Liu, P. D.; Zhang, X. 2010. The study on multi-attribute decision-making with risk based on linguistic variable, International Journal of Computational Intelligence Systems 3(5): 601-609.

Liu, P. D.; Zhang, X. 2011a. Investigation into evaluation of agriculture informatization level based on two-tuple, Technological and Economic Development of Economy 17(1): 74-86. http://dx.doi.org/10.3846/13928619.2011.554007

Liu, P. D.; Zhang, X. 2011b. Research on the supplier selection of supply chain based on entropy weight and improved ELECTRE-III method, International Journal of Production Research 49(3): 637-646. http://dx.doi.org/10.1080/00207540903490171

Liu, P. D.; Zhang, X.; Liu, W. L. 2011. A risk evaluation method for the high-tech project investment based on uncertain linguistic variables, Technological Forecasting and Social Change 78(1): 40-50. http://dx.doi.org/10.1016/j.techfore.2010.04.011

Wei, G. W.; Huang, D. S.; Wei, Y. 2007. ULHGA operator and its application to group decision making under uncertain linguistic environment, Fuzzy System and Mathematics 21(2): 72-78.

Xu, X. Q.; Cheng, J. M.; Xu, M. X. 2007. A projection model based on linguistic term indices in uncertain linguistic multi-attribute decision making, Value Engineering (5): 127-130.

Xu, Z. S. 2004a. Uncertain Multi-attribute Decision Theory and Its Application. Beijing: Tsinghua University Press.

Xu, Z. S. 2004b. Uncertain linguistic aggregation operators based approach to multiple attribute group decision making under uncertain linguistic environment, Information Science 168(1-4): 171-184. http://dx.doi.org/10.1016/j.ins.2004.02.003

$\mathrm{Xu}, \mathrm{Z}$. S. 2004c. EOWA and EOWG operators for aggregating linguistic labels based on linguistic preference relations, International Journal of Uncertainty, Fuzziness and Knowledge-Based Systems 12(5): 791-810. http://dx.doi.org/10.1142/S0218488504003211

$\mathrm{Xu}, \mathrm{Z}$. S. 2005. Deviation measures of linguistic preference relations in group decision making, Omega 33(3): 249-254. http://dx.doi.org/10.1016/j.omega.2004.04.008

$\mathrm{Xu}, \mathrm{Z}$. S. 2006a. Induced uncertain linguistic OWA operators applied to group decision making, Information Fusion 7(2): 231-238. http://dx.doi.org/10.1016/j.inffus.2004.06.005

$\mathrm{Xu}, \mathrm{Z}$. S. 2006b. Minimizing deviations models for solving MADM problems with preference information on alternatives in uncertain linguistic setting, International Journal of Operations Research 3(1): 30-35.

$\mathrm{Xu}, \mathrm{Z}$. S. 2006c. A direct approach to group decision making with uncertain additive linguistic preference relations, Fuzzy Optimization and Decision Making 5(1): 23-35.

http://dx.doi.org/10.1007/s10700-005-4913-1

Xu, Z. S. 2006d. An approach based on the uncertain LOWG and the induced uncertain LOWG operators to group decision making with uncertain multiplicative linguistic preference relations, Decision Support Systems 41(2): 488-499. http://dx.doi.org/10.1016/j.dss.2004.08.011

$\mathrm{Xu}, \mathrm{Z}$. S. 2008a. Theory and Methods of Decision Making with Linguistic Information. Beijing: Science Press, 147-148.

$\mathrm{Xu}, \mathrm{Z}$. S. 2008b. Group decision making based on multiple types of linguistic preference relations, Information Sciences 178(2): 452-467. http://dx.doi.org/10.1016/j.ins.2007.05.018

$\mathrm{Xu}, \mathrm{Z}$. S. 2009. An interactive approach to multiple attribute group decision making with multigranular uncertain linguistic information, Group Decision and Negotiation 18(2): 119-145.

http://dx.doi.org/10.1007/s10726-008-9131-0 
$\mathrm{Xu}, \mathrm{Z}$. S. 2010. Interactive group decision making procedure based on uncertain multiplicative linguistic preference relations, Journal of Systems Engineering and Electronics 21(3): 408-415.

Yan, M. R.; Pong, C. S.; Lo, W. 2011. Utility-based multicriteria model for evaluating BOT projects, Technological and Economic Development of Economy 17(2): 207-218. http://dx.doi.org/10.3846/20294913.2011.580585

Yager, R. R. 2004. OWA aggregation over a continuous interval argument with applications to decision making, IEEE Transactions on Systems, Man, and Cybernetics. Part B, Cybernetics 34(5): 1952-1963. http://dx.doi.org/10.1109/TSMCB.2004.831154

Zeng, X. B. 1997. New study on comprehensive weight method, Forecasting (5): 69-72.

Zhang, H. M.; Xu, Z. S. 2005. Uncertain linguistic information based C-OWA and C-OWG operators and their applications, Journal of PLA University of Science and Technology 6(6): 604-608.

Zhang, N. C.; Li, Z. L.; Liu, C. Y. 2007. Choice of landing area based on uncertain linguistic information multiple attribute decision making, Ship Electronic Engineering 27(5): 53-56.

Zhang, S.; Wang, Y. L.; Gao, Y. 2006. A kind of method for multiple attribute group decision making based on uncertain linguistic information and its application, Journal of Air Force Engineering University (Natural Science Edition) 7(6): 47-50.

Zhou, H. A.; Liu, S. Y. 2007. Projection method of fuzzy multi-attribute decision-making based on the maximal deviation model, Systems Engineering and Electronics 29(5): 741-744.

Zhu, W. D.; Zhou, G. Z.; Yang, S. L. 2009. An approach to group decision making based on 2-dimension linguistic assessment information, Systems Engineering 27(2): 113-118.

Peide LIU (China, 1966) obtained the bachelor degree and master degree in electronic technology in the Southeast University, and obtained doctor degree in information management in Beijing Jiaotong University. Now he is a full-time professor in Shandong University of Finance and Economics and assistant director of the Enterprise's Electronic-commerce Engineering Research Center of Shandong. His main research fields are technology and information management, decision support and electronic-commerce. 